\title{
DISEÑO DE UN PROGRAMA PSICOPEDAGÓGICO PARA LA INTERVENCIÓN EN LOS TRASTORNOS EMOCIONALES EN LA ENSEÑANZA Y APRENDIZAJE DE LAS MATEMÁTICAS
}

\author{
Eloísa Guerrero Barona y Lorenzo J. Blanco Nieto \\ Universidad de Extremadura
}

\section{INTRODUCCIÓN}

La educación matemática viene condicionada por múltiples factores que han sido considerados en mayor o menor medida en diferentes investigaciones educativas. En los últimos años hemos constatado un aumento de publicaciones que relacionan la dimensión afectiva del individuo (creencias, actitudes y emociones) y la enseñanza/aprendizaje de las matemáticas (Gairín, 1990; Miranda, Fortes y Gil, 1998, Mceod, 1992 y 1994, Schoenfeld, 1992, Gómez-Chacón, 1997, 1999, 2000; Blanco y Guerrero, 2002 y Guerrero y Blanco, 2002).

La formación del alumnado en el ámbito emocional y afectivo se fundamenta en la importancia que tienen nuestros pensamientos y creencias en la explicación del modo en que nos comportarnos ante las actividades matemáticas. Estos ámbitos explican los rechazos y las atracciones hacia las mismas, hacia el profesorado que la enseña, hacia la situación de aprendizaje en la que se desarrolla, y, en general, hacia la escuela, hacia los demás o hacia ellos mismos. La diversidad y la variedad emocional, que tanto profesores como alumnos pueden experimentar, influirán de manera decisiva sobre la salud física y/o emocional de ambos.

En este artículo tratamos, en primer lugar, los binomios emociones y rendimiento, las actitudes y creencias en la educación matemática y cómo aprender/enseñar a resolver problemas. En segundo lugar, proponemos el diseño de un programa de intervención psicopedagógica para el entrenamiento en la resolución de problemas y en competencias personales y emocionales.

\section{EMOCIONES Y RENDIMIENTO}

La mejor manera de explicar cómo los estados emocionales influyen en nuestro rendimiento es a partir de la premisa siguiente: los pensamientos, las creencias y las actitudes determinan los sentimientos y las emociones. Es decir, no son los hechos reales, sino el significado y las evaluaciones que el sujeto realiza las que producen cambios en las emociones y en los estados de ánimo. Las experiencias son procesadas y reciben un significado antes de experimentar una respuesta emocional como efecto de las evaluaciones de los sucesos internos o externos (Lazarus, 1991; Lewis y Havilland, 1993; Oattley y Jenkins, 1996). Este hecho lleva al individuo a buscar una explicación o etiquetaje que determinará la respuesta emocional (alegría, miedo, frustración, tristeza, etc.). En consecuencia, la emoción depende del pensamiento y el pensamiento precede a la emoción. Si podemos cambiar las evaluaciones, interpretaciones y atribuciones, también podremos cambiar las emociones de miedo y la ansiedad que experimenta. Cuando una persona está ansiosa se está interpretando los sucesos como amenazantes y peligrosos, creándose un circuito de retroalimentación negativa entre nuestros pensamientos y la actividad psicofisiológica. Como resultado, aparecen valoraciones y pensamientos derrotistas y catastrofistas: me voy a bloquear, perderé los papeles, es muy difícil, haré el ridículo, es un rollo, etc. 
En la última mitad de los años 90 ha aumentado el número de estudios y publicaciones relacionadas con las emociones (Lazarus, 1991; Lewis y Havilland, 1993; Oattley y Jenkins, 1996; Fernández-Abascal y Palmero, 1999 y Nezu, Nezu y Blissett, 1988), siendo la ansiedad una de las que más literatura científica está generando por su relación con la salud.

El sistema educativo ha estado tradicionalmente más interesado en enseñar conocimientos que en la Psicopedagogía de la emociones. Bisquerra (1999), recientemente ha señalado que una de las manifestaciones más comunes de analfabetismo emocional son los trastornos psicopatológicos, entre los que se encuentra la ansiedad. La ansiedad puede conceptualizarse, bien como un estado emocional crónico, manifestando sus efectos en cualquier tipo de situación (rasgo), bien como la susceptibilidad a presentar reacciones emocionales, sólo en determinadas situaciones (estado) (Bermúdez, 1986).

El término "emociones negativas" ha cobrado fuerza en los últimos años y se refiere a las emociones que producen una experiencia emocional desagradable, como la ira, la tristeza y la ansiedad (Cano-Vindel y Miguel-Tobal, 2001).

Tobal (1985), Cano Vindel y Miguel-Tobal (2001) entienden la ansiedad como una respuesta o patrón de respuestas que engloba aspectos cognitivos displacenteros, de tensión y aprensión; aspectos fisiológicos, caracterizados por un alto nivel del sistema nervioso autónomo, y aspectos motores que suelen implicar comportamientos poco ajustados y escasamente adaptativos. Esta respuesta puede ser elicitada, tanto por situaciones o estímulos externos, como por estímulos internos, tales como pensamientos, creencias, expectativas, atribuciones, etc., que son percibidos por el sujeto como peligrosos.

Las situaciones en las que se desencadena la reacción de ansiedad tienen en común la previsión de posibles consecuencias negativas o amenazantes para el sujeto. Esta reacción supone una puesta en marcha de diferentes recursos cognitivos (atención, memoria, percepción, pensamiento, lenguaje, etc.), fisiológicos (activación autonómica, motora, etc.) y conductuales (alerta, evitación etc.).

Cuando un individuo mantiene altos niveles de ansiedad, durante tiempos prolongados, su bienestar psicológico se ve seriamente perturbado; sus sistemas fisiológicos pueden verse alterados por un exceso de actividad, su sistema inmune puede verse incapaz de defenderse y sus procesos cognitivos pueden verse alterados, provocando una disminución del rendimiento, la evitación de situaciones que provocan reacciones de intensa activación puede afectar a la vida personal, académica y social (Cano-Vindel y Miguel-Tobal, 2001). La actividad cognitiva superior puede verse afectada por procesos emocionales con los que mantiene una estrecha relación. Así, por ejemplo, el rendimiento en los exámenes o en otra situación de evaluación, como la tarea matemática, puede verse deteriorada cuando el sujeto se ve desbordado por su reacción de ansiedad.

La relación entre activación y rendimiento ha sido ampliamente estudiada a través de diversas teorías: teoría del 'drive' o activación del organismo (Spence y Spence, 1966), la hipótesis de la U invertida (Yerkes y Dodson, 1980), la teoría de la zona de funcionamiento óptimo (Hanin, 1980), el modelo de catástrofe (Hardy, 1990) y la teoría de cambio (Kerr, 1985). La primera asume que el rendimiento es mayor cuanto mayor es el 'drive' o activación del organismo, es decir, hay una asociación directa y lineal entre activación y rendimiento. La limitación de esta teoría es que sólo es aplicable para tareas motoras y mecánicas simples. Este hecho ha dado paso a una teoría explicativa más completa, la teoría de la $U$ invertida, según la cual el rendimiento aumentará en la medida en que también aumente el nivel de 
activación, aunque sólo hasta un punto, a partir del cual, el aumento de la activación propiciará una disminución del rendimiento. Según esta hipótesis, tanto la ausencia o nivel bajo de activación, como la activación demasiado elevada perjudicarán el rendimiento, mientras que un cierto nivel de activación lo favorecerá notablemente.

Diferentes investigaciones han constatado resultados contradictorios debido a la variable "diferencias individuales", en el sentido de que existen importantes sesgos que determinan la evaluación de las situaciones potencialmente estresantes y de los propios recursos del sujeto para hacerles frente. Lo que sí parece evidente es que cada individuo tiene una zona de activación óptima que favorece el máximo rendimiento, (teoría de la zona de funcionamiento óptimo) y que por encima y por debajo de ésta, el rendimiento es más deficitario. Habría que estudiar cuál es la relación particular entre activación y rendimiento de cada sujeto en concreto.

La teoría de la catástrofe de Harhy (1990) sugiere que la ansiedad somática (hiperactivación fisiológica) y el rendimiento pueden tener una relación $U$ invertida, siempre que la ansiedad cognitiva (distorsiones pensamientos y creencias) esté ausente, pues en su presencia provocará una situación desfavorable (catástrofe) que interrumpirá el continuo curvilíneo y perjudicará el rendimiento. Es importante la distinción entre activación fisiológica y activación cognitiva, y entre la activación "positiva" y "negativa", de manera que la activación negativa provoca un déficit de rendimiento por exceso de activación, aunque en pequeñas dosis puede resultar útil.

Finalmente, la teoría del cambio señala que la activación será beneficiosa o perjudicial en función de la evaluación e interpretación que el sujeto haga de la misma (Lazarus y Folkman, 1976). Según esta teoría, una activación elevada será favorable si es interpretada como excitación placentera (motivación) y perjudicial, si la interpreta como ansiedad (estrés). De igual modo, una baja activación será favorable si es interpretada como relajación (ausencia de estrés), pero desfavorable si la interpreta como aburrimiento)(estrés). De acuerdo con esta teoría, el problema puede solucionarse cambiando la interpretación a través de la intervención cognitiva.

De las teoría anteriores se pueden extraer algunas conclusiones, como por ejemplo, que un alto grado de ansiedad facilita el aprendizaje mecánico, pero tiene efecto inhibitorio sobre los tipos de aprendizaje más complejos, que son menos familiares o que dependen más de habilidades de improvisación que de persistencia. En relación a las Matemáticas, Gairín (1991) ha señalado que la ansiedad facilita el aprendizaje de tareas complejas cuando se dan las siguientes circunstancias: a) no amenazan la autoestima personal, b) no son tareas exageradamente novedosas o significativas, c) la ansiedad es sólo moderada, y, d) cuando el estudiante posee mecanismos efectivos de superación de la ansiedad. Los estudiantes con un alto nivel de ansiedad se benefician más de las lecciones expositivas, mientras que los estudiantes con un bajo nivel de ansiedad se benefician más de los métodos de aprendizaje por descubrimiento. Recordemos, que las nuevas propuestas curriculares huyen del aprendizaje mecánico y memorístico, así como de las clases expositivas para buscar una enseñanza donde la participación, colaboración, comunicación, etc. permitan la construcción del conocimiento matemático. 


\section{ACTITUDES Y CREENCIAS EN MATEMÁTICAS}

Un comportamiento ajustado tendrá más posibilidades de llevarse a cabo, si previamente se apoya en unas actitudes funcionales y éstas a su vez se basan en unos conocimientos específicos y racionales sobre tales conductas. Las conductas adaptadas sólo son posibles si existe una motivación y unas actitudes que facilitan que el comportamiento se lleve a cabo. De ahí la importancia que otorgamos al proceso actitudinal. Entendemos por actitud la predisposición permanente conformada de acuerdo a una serie de convicciones y sentimientos que hacen que el sujeto reaccione (favorable o desfavorablemente), tienda a expresarse en actos y opiniones ante una situación, objeto o persona, de acuerdo con sus creencias y sentimientos. Es un modo de estar respecto de algo o alguien, un compuesto relativo a lo que el individuo piensa, siente y hace respecto a un determinado objeto sociocultural (Newcomb, 1976).

En relación con las matemáticas, Gómez-Chacón (2000) considera que las actitudes constan de tres componentes: cognitivo, que se manifiesta en las creencias subyacentes a dicha actitud, afectivo que se expresa en sentimientos de aceptación o de rechazo de la tarea o de la materia, y, por último, un componente intencional o de tendencia a un cierto tipo de comportamiento. En definitiva, las actitudes se concretan y se expresan en ideas y creencias, en sentimientos hacia objetos y personas y modos de actuar específicos.

Las actitudes que los estudiantes van generando como producto de su experiencia escolar hacia las matemáticas se van estabilizando y haciéndose resistentes a los cambios, conforme avanzan en niveles educativos (Gairín, 1990). Callejo (1994) hace una clara distinción entre actitudes matemáticas y actitudes hacia la matemática. Las actitudes matemáticas tendrán un marcado componente cognitivo y se refieren al modo de utilizar las capacidades generales que son importantes en el trabajo matemático. Las actitudes hacia la matemática aluden a la valoración, aprecio e interés por la materia y por su aprendizaje, predominando el componente afectivo. Rechazo, negación, frustración, pesimismo y evitación son algunas de las manifestaciones actitudinales y comportamentales de muchos alumnos cuando afrontan la tarea matemática (Guerrero, Blanco y Vicente, 2002). Además, como consecuencia de su experiencia escolar los estudiantes van generando creencias acerca de la matemática (el objeto), acerca de la enseñanza/aprendizaje de la Matemática, y creencias acerca de uno mismo en relación con la educación matemática. Estas últimas tienen una fuerte componente afectivo que engloba las relacionadas con la confianza en uno mismo, su autoconcepto y la autoeficacia percibida.

Como resultado de la actividad desarrollada en relación a la resolución de problemas, los estudiantes adquieren una concepción sobre los problemas matemáticos, sobre la forma de resolverlos, sobre el papel de la enseñanza de las matemáticas que va a provocar en ellos actitudes concretas para abordarlos. El fracaso continuado ante procesos, normalmente mecánicos y repetidos, sobre resolución de problemas (problemas tipos) siguiendo procedimientos algorítmicos, elicita en ellos una actitud negativa hacia la resolución de problemas. De igual manera, la falta de recursos para esolver problemas más complejos les lleva a una baja autoestima como resolutores de problemas y a la consideración de que los buenos resolutores son los estudiantes más listos (Blanco, 1997; Gómez-Chacón, 1997). Probablemente, como efecto de su historia repetida de fracasos dudan de su capacidad intelectual y llegan a considerar sus esfuerzos inútiles. De ahí el sentimiento de frustración y el deseo de abandonar rápidamente ante la dificultad. Esto determina que surjan nuevos fracasos que reforzarán la creencia de que efectivamente son 
incapaces de lograr el éxito. Esta situación les lleva a asumir una responsabilidad menor sobre sus éxitos, lo que puede a su vez producir indefensión aprendida (Miranda, Fortes y Gil, 1998).

Muchos estudiantes mantienen la idea de que las Matemáticas desarrollan el razonamiento lógico, contribuyendo así a la formación de cada persona. Esto apunta a una concepción de las Matemáticas como ciencia por excelencia que favorece la formación intelectual del individuo. Sin embargo, al relacionar esta creencia con la experiencia académica aparece la "imagen" de los mejores alumnos en clase de matemáticas, que suponen son los más preparados y más inteligentes del grupo. La combinación de esta idea e imagen puede acompañarse de expresiones y autoverbalizaciones como: "las Matemáticas siempre han sido complicadas y trabajosas" o "son un rompecabezas", y así, es como el alumno elabora la creencia de "las Matemáticas como ciencia abstracta, rigurosa, exacta, lógica". En relación a esto último, piensan los alumnos, aunque no lo explicitan, que las Matemáticas son inaccesible para muchos, provocando una baja autoestima y un deterioro en la autoeficacia, en relación a la actividad matemática y como resolutores de problemas.

Todas estas creencias pueden llevar al alumno a exagerar la importancia de obtener resultados exitosos y a subestimar su propia valía. Su incapacidad para resolver problemas se convierte en algo totalmente angustioso, puesto que toda su persona se siente amenazada. De esta manera, el hecho de que se les plantee un problema para su resolución se convierte en una situación angustiosa que desencadena niveles elevados de ansiedad de los que desea a toda costa escapar, abandonando la situación.

A través de un procedimiento de reforzamiento negativo se mantienen sus creencias y se explica el proceso evitativo. En otras palabras, se refuerza la creencia de que es incapaz de resolver problemas, por lo que cuando se vuelva a enfrentar a una tarea matemática lo hará con niveles aún mayores de ansiedad, ya que ahora tiene más pruebas de su incompetencia. Todo ello hará que aumente la probabilidad de responder de nuevo abandonando la situación una y otra vez (Miranda, Fortes y Gil, 1998).

Las respuestas de los alumnos ante esta situación son muy diversas. En la figura 1 aparecen agrupadas algunas de ellas, obtenidas a partir de registros de observaciones en el aula (Guerrero, Blanco y Vicente, 2002).

\section{Figura 1}

Manifestaciones en los tres sistemas de respuesta de la ansiedad ante la tarea matemática

\begin{tabular}{|c|c|c|}
\hline $\begin{array}{l}\text { Respuestas cognitivas } \\
\text { ("Pensamos") }\end{array}$ & $\begin{array}{l}\text { Respuestas fisiológicas y } \\
\text { emocionales ("Sentimos") }\end{array}$ & $\begin{array}{l}\text { Conductas } \\
\text { ("Hacemos") }\end{array}$ \\
\hline $\begin{array}{l}\text { "Es muy difícil" } \\
\text { "No voy a entenderlo" } \\
\text { "Es un rollo" } \\
\text { "Mejor dejarlo" } \\
\text { "Esto es sólo para listos" } \\
\text { "El profesor me reñirá" } \\
\text { "Esto es una comedura de coco" }\end{array}$ & $\begin{array}{l}\text { Sentimientos de impotencia, } \\
\text { fracaso e inutilidad } \\
\text { Miedo, preocupación y/o } \\
\text { irritabilidad } \\
\text { Tensión muscular } \\
\text { Sudoración, sonrojo o rubor } \\
\text { Nudo en la garganta }\end{array}$ & $\begin{array}{l}\text { Repetir una y otra vez el inicio de } \\
\text { la tarea } \\
\text { Evitación y escape } \\
\text { Abandonos } \\
\text { Interrumpir la tarea } \\
\text { Tocarse, rascarse,... }\end{array}$ \\
\hline
\end{tabular}

\section{APRENDER/ENSEÑAR A RESOLVER PROBLEMAS}

Uno de los objetivos principales en educación matemática es que los alumnos sean capaces de desarrollar y aplicar estrategias para la resolución de problemas. A este respecto, las orientaciones curriculares señalan que "el alumno debe desarrollar y perfeccionar sus propias estrategias, a la vez que 
adquiera otras generales y específicas que le permiten enfrentarse a las nuevas situaciones con probabilidad de éxito"(MEC, 1992, 92). En este sentido, se ha de ofrecer a los alumnos la oportunidad de familiarizarse con los procesos que facilitan la exploración y resolución de problemas como comprensión y expresión de la situación matemática (verbalización, dramatización, discusión en equipo), extracción de datos y análisis de los mismos, representación en forma gráfica del problema o situación, formulación de conjeturas y verificación de su validez o no, exploración mediante ensayo y error, formulaciones nuevas del problema, comprobación de resultados y comunicación de los mismos. Además se hace necesario desarrollar la capacidad de persistir en la exploración de un problema.

La experiencia nos muestra que algunos estudiantes, como resultado de su aprendizaje matemático, llegan a ser buenos resolutores de problemas. Lo que no parece estar tan claro es si se puede enseñar a resolver problemas, es decir, que se pueda enseñar técnicas o estrategias que garanticen la resolución correcta de todo tipo de problemas.

Experiencias e investigaciones desarrolladas con alumnos y profesores de diferentes niveles han intentado dar respuesta al interrogante anterior, planteando esquemas de trabajo de cómo podría hacerse. También se ha intentado conocer cuál es el comportamiento de los resolutores de problemas (Guzmán, 1991; Callejo, 1994; Puig, 1996; Carrillo, 1998). Es evidente que tales experiencias no pueden ser trasplantadas al aula, pero contienen valiosas recomendaciones que ayudan en la actividad docente a cumplir el objetivo señalado.

Una de las ideas que apuntamos es que el desarrollo de la capacidad de resolver problemas no puede ser un objetivo alcanzable fuera de un ambiente de resolución de problemas. Además, es conveniente fomentar un proceso de reflexión y comunicación de ideas e intenciones, tanto durante el proceso de resolución, como una vez concluido el mismo, ya que es de suma importancia por las siguientes razones: 1) porque una de las condiciones básicas para que se produzca aprendizaje en procesos tan complejos como la resolución de problemas es facilitar un clima de libertad para animar a los alumnos a expresar sus ideas 2) porque conocer mejor el funcionamiento de la mente de otras personas es un modo de mejorar la habilidad para resolver problemas por dos motivos principales: primero, porque se pueden descubrir nuevas formas de aproximarse al problema, de percibirlo, de afrontarlo; segundo, porque permite constatar que los "demás" también tienen bloqueos, vacilaciones, fallos, etc., lo que debilita el miedo a fracasar ante esta tarea, $y, 3$ ) porque tratar de convencer a otros miembros del grupo de que una conjetura es cierta es una de las formas de experimentar la necesidad de la demostración en el dominio matemático (Callejo, 1994).Estas razones, que son importantes, deben ser asumidas por los resolutores, y para ello es necesario que vivan su propia experiencia. Creemos que cuando los alumnos colaboran en grupo resuelven problemas que no resolverían si trabajasen solos. Una sugerencia de actuación apuntada por uno de ellos, se puede debatir y reformularla en el grupo, dando lugar a un camino de solución. Los estudiantes participan juntos en un diálogo interno al grupo similar al que desarrollan consigo mismo los buenos resolutores de problemas. Esta comunicación que se establece entre ellos, o consigo mismo, resolviendo el problema es la base que permite reflexionar y discutir sobre el proceso seguido, favoreciendo que los resolutores puedan comprender y asimilar los diferentes procedimientos para resolver problemas. Consideramos que este paso es esencial para el aprendizaje de estrategias de resolución de problemas, porque facilita a los estudiantes hablar acerca del proceso de resolución, más que preguntar la respuesta, encontrándonos con comentarios personales inesperados que permiten al profesor conocer más a los 
estudiantes y a éstos les permite conocerse a si mismos. Es importante advertir que este proceso de reflexión no debe confundirse con la descripción del conjunto de operaciones con el que se obtiene el resultado a partir de los datos.

La experiencia ha demostrado que esta metodología ayuda a ver las capacidades de los estudiantes. Igualmente, contribuye a que los estudiantes valoren mejor la resolución de problemas, encuentren justificación para un mayor y más organizado esfuerzo y posean una visión más amplia de las matemáticas. Comprendemos que no se puede garantizar el éxito al instante o ante cualquier problema, ni siquiera es el principal objetivo. Sí en cambio, estamos convencidos de que es posible proporcionar experiencias que faciliten destrezas útiles para abordar la resolución de problemas con mayor autoestima y esperanza, evitando la desesperación inicial y la desconfianza cuando los caminos son inciertos. Se pueden proponer problemas sugerentes, despertar el interés por la actividad matemática, dar pautas e indicaciones, ayudar a los estudiantes a explicitar sus procesos de pensamiento y a reflexionar sobre ellos, etc. Pero, la manera de abordar la resolución de problemas es algo muy personal y en este sentido lo que se puede hacer es ayudar a cada estudiante a descubrir su propio estilo, sus capacidades y sus limitaciones (Callejo, 1994).

Como conclusión de todo ello, no se trata pues de transmitir a los estudiantes, solamente, métodos, reglas heurísticas o trucos, sino de abordar las actitudes y las creencias que han conducido a estos procedimientos, partiendo de sus propias experiencias e historias de aprendizaje. En esta segunda parte del artículo proponemos el diseño de un programa de intervención para el entrenamiento en resolución de problemas y en competencias emocionales, basado en la responsabilidad del alumno y en el conocimiento de sus propios procesos cognitivos, emocionales y afectivos para superar las dificultades. Detallamos los elementos que lo conforman, partiendo de la hipótesis principal. Describiremos los objetivos, la metodología, la secuencia de su desarrollo y la explicación del programa sesión a sesión.

\section{PROPUESTA DE UN DISEÑO DE INTERVENCIÓN PSICOPEDAGÓGICA Hipótesis}

Tras la revisión anterior, partimos de la siguiente hipótesis: las actitudes, las creencias, los pensamientos, los conocimientos y las emociones de los alumnos determinan el éxito y/o el fracaso ante las matemáticas.

\section{Objetivos}

Tras previa evaluación de las actitudes, las creencias y la valoración del patrón de respuestas de ansiedad de los alumnos, nos proponemos que el alumno alcance los objetivos siguientes: 1) aprender a resolver problemas, a través de la puesta en práctica de una metodología de enseñanza/ aprendizaje basada en un modelo de resolución de problemas, 2) disminuir el estado de activación y tensión psicofisiológica que pueda interferir negativamente en el rendimiento, a través del entrenamiento en relajación y en control de la respiración y 3) enseñar y entrenar en autoinstrucciones funcionales que permitan manejar pensamientos y emociones ante la tarea matemática, al mismo tiempo que perciban la situación como no amenazante.

\section{Método}




\section{Sujetos}

El grupo de trabajo lo formarán de 10 a 15 alumnos y las sesiones tendrán una duración de $1 \mathrm{~h}$, aproximadamente. Está diseñado para ser aplicado a alumnos a partir del tercer ciclo de educación primaria hasta bachillerato, y para ser trabajado en la formación inicial y prmanente del profesorado.

\section{Instrumentos de evaluación}

Para la evaluación de actitudes, afectos y creencias proponemos emplear los cuestionarios empleados por Gómez Chacón (2000). Especialmente útiles son los cuestionarios que hacen alusión a las opiniones sobre las matemáticas y a la conexión entre el alumno y las matemáticas de esta misma obra. También son de utilidad el cuestionario de Callejo (1994) sobre concepciones de los alumnos sobre resolución de problemas, y el de Camacho (1995) acerca de comportamientos y actitudes hacia las matemáticas y su enseñanza.

Para la evaluación de los trastornos de ansiedad, del estado del ánimo, del autoconcepto, del autocontrol y de la personalidad, proponemos los siguientes cuestionarios:

- S.T.A.I.C, Cuestionario de Autoevaluación Ansiedad Estado/Rasgo en niños (Spielberger y col, 1990). Es una prueba que ofrece dos evaluaciones: evaluación ansiedad como estado transitorio, fruto quizá de una situación frustrante o problemática y la ansiedad como rasgo permanente en la dinámica personal del sujeto. Aplicable en niños de 9 a 15 años. En lo que respecta a la fiabilidad, aunque los coeficientes encontrados no son elevados, pueden considerarse bastante satisfactorios para un instrumento de medida relativamente corto (con sólo 20 elementos) obteniéndose por el método de las dos mitades corregidos los resultados 0,91 y 0,87 para A-E y A-R respectivamente.

- C.A.C.I.A., Cuestionario de Autocontrol Infantil y Adolescente, (Bonet y Silva, 1986). Nos permite valorar la escala de autocalifacción en tres dimensiones de autocontrol positivo (retroalimentación personal, retraso de la recompensa y autocontrol), una dimensión de autocontrol negativo (autocontrol procesual) y una escala de sinceridad. Es aplicable a niños entre los 11 y 19 años.

- C.D.S., Cuestionario de Depresión para Niños (Lang y Tisher, 1986), especialmente, las subescalas de autoestima, de respuesta afectiva. Permite la valoración global y específica de la depresión en niños y es aplicable a niños entre los 8 y 16 años.

- C.P.Q., Cuestionario de Personalidad para Niños (Porter y Cattell, 1988). Nos permite valorar 14 rasgos de primer orden y 3 de segundo orden de la personalidad y es aplicable a niños entre los 8 y 12 años. Otros cuestionarios elaborados por estos mismos autores para distintos rangos de edades son: HSPQ (12 y 18 años) y el ESPQ (6-8 años). El primero se aplica en sujetos de 12 a 18 años y el segundo a ñiños de edades entre 6 a 8 años. En general son útiles para la valoración general de la adaptabilidad escolar, especialmente en la etapa primaria.

\section{Procedimiento}

El programa está diseñado para ser desarrollado en un Taller-Seminario denominado: "Mejora de nuestras competencias personales y emocionales ante las matemáticas". La finalidad es aprender a resolver 
problemas y desarrollar habilidades que permitan a los alumnos afrontar situaciones ansiógenas ante las matemáticas. La duración será de un mes y medio y se estructura en 10 sesiones.

El programa está inspirado en el modelo de Polya (1985) sobre resolución de problemas y en el modelo de inoculación de estrés (Meicchenbaum, 1985). El primero enseña a resolver problemas y a entrenar los procesos cognitivos implicados (Blanco, 1991, Guzman, 1991, Callejo, 1994 y Carrillo, 1996), y el segundo, adiestra al alumno a afrontar situaciones generadoras de ansiedad, a relajarse fisiológicamente y a manejar sus emociones.

El modelo de resolución de problemas consta de cuatro fases, y para cada una de ellas se sugieren una serie de ${ }^{1}$ heurísticos que sirven de guía en el proceso. Ayudan al resolutor a aproximarse y comprender el problema, y a ordenar eficientemente sus recursos para resolverlo. Al referirnos a los heurísticos, no queremos establecer una relación de acciones a modo de pruebas de entrenamiento, puesto que no tratamos de transmitir a los estudiantes reglas heurísticas o trucos para la resolución de problemas, sino ayudarles a descubrir su propio estilo, sus capacidades y sus limitaciones, pero diseñando actividades que favorezcan hábitos adecuados de resolución de problemas.

El modelo de inoculación de estrés consta de cuatro fases y permite relajar la activación fisiológica y sustituir pensamientos, creencias y actitudes negativas por pensamientos funcionales.

Figura 2

Modelos procesuales del programa de intervención

Fases del modelo de resolución de problemas: Analizar y comprender el problema, buscar estrategias de solución, llevar a cabo el plan, revisión de la solución y del proceso

Fases del modelo de inoculación de estrés: Entrenamiento en relajación y control de respiración, reestructuración cognitiva, la resolución del problema y entrenamiento en autoinstrucciones.

\section{DESARROLLO, SECUENCIA Y EXPLICACIÓN DE LAS SESIONES}

Tras realizar la evaluación pretratamiento, en la primera sesión se presenta el programa y se explica la fundamentación de los dos modelos en los que se basa. Se expone y se discute en grupo la hipótesis de partida (Sesión introductoria o educativa).

En la segunda y tercera sesión, durante la primera $1 / 2 \mathrm{hora}$, iniciamos el entrenamiento en control de respiración y relajación. Recomendamos su práctica fuera del entorno académico. El tiempo restante abordamos el primer paso del modelo de resolución de problemas: analizar y comprender un problema. Se trata de que el alumno se familiarice con la situación planteada en el problema, y para lograrlo sugerimos organizar la información imaginando mentalmente la situación, releyendo el enunciado para interiorizar la situación con datos y objetivos, utilizando algún modelo manipulativo y/o algún esquema gráfico e introduciendo alguna notación que nos permita simplificarla y ordenarla.

En la cuarta sesión se inicia la preparación para afrontar la ansiedad y las emociones negativas. Consiste en centrarse, en combatir pensamientos negativos e insistir en la importancia de la planificación y la preparación. Una de las tareas en esta sesión es la elaboración de un listado de estresores y de autoinstrucciones funcionales (véase figura3). Las autoinstrucciones funcionales son útiles para controlar

\footnotetext{
${ }^{1}$ Los heurísticos son sugerencias concretas encuadradas en el proceso general que ayudan al resolutor a desarrollar habilidades y actitudes positivas en el proceso de resolución. Las actividades sugeridas son usadas por buenos resolutores de problemas cuando necesitan comprender una situación o hacer progresos hacia la solución o analizar el proceso seguido.
} 
la cadena de pensamientos distorsionados porque actuarán como un interruptor del circuito de las emociones negativas, comunicando al cuerpo que no hay necesidad de activación fisiológica y que puede relajarse. No se trata de sustituir sólo autoafirmaciones negativas por positivas, sino de lograr esta sustitución a través de la modificación de las creencias.

En lo referente a la resolución de problemas, a partir de la quinta sesión, iniciamos la fase de búsqueda de estrategias de solución de problemas. Recurriremos a nuestros conocimientos y experiencia, lanzando las preguntas, como las que aparecen recogidas en el punto 2 de la figura 3.

\section{Figura 3}

Modelo de resolución de problemas y entrenamiento en autoinstrucciones

\begin{tabular}{|c|c|}
\hline Modelo de resolución de problemas & Entrenamiento en autoinstrucciones \\
\hline $\begin{array}{l}\text { 1. Analizar y comprender el problema } \\
\text { ¿Qué es lo que desconoces?, ¿cuáles son los } \\
\text { datos? ¿cuáles son las condiciones?, ¿es } \\
\text { posible cumplir las condiciones del problema?, } \\
\text { ¿son suficientes, insuficientes, contradictorios } \\
\text { para cumplir los objetivos el problema?, ¿qué } \\
\text { conceptos y procesos matemáticos están } \\
\text { implicados en } \\
\text { el problema?, ¿los dominas?, } \\
\text { 2. Buscar una estrategia de solución } \\
\text { ¿Has visto este problema anteriormente, otro } \\
\text { igual o parecido?, ¿conoces alguno } \\
\text { relacionado, algún teorema que pueda ser } \\
\text { útil?. observando el planteamiento del } \\
\text { problema, intenta pensar sobre problemas que } \\
\text { tengan la misma o similar incógnita. en estas } \\
\text { condiciones, ¿hay algún problema que has } \\
\text { resuelto?, ¿podrías usarlo?, ¿podrías usar su } \\
\text { resultado o su método? } \\
\text { 3. Llevar a cabo el plan y examen } \\
\text { Comprobar que los pasos son correctos. } \\
\text { Registrar todos los cálculos, resaltar los logros } \\
\text { intermedios, actuar con orden, con precisión y } \\
\text { explicar el estado de la ejecución. } \\
4 . \text { Revisión de la solución y del proceso } \\
\text { Haremos al alumno las siguientes preguntas: } \\
\text { ¿sabes analizar el resultado, examinar los } \\
\text { argumentos?, ¿sabes obtener estos } \\
\text { resultados de diferente modo?, ¿podría } \\
\text { resolverlo de un vistazo?, ¿puede usar el } \\
\text { resultado para otro problema? }\end{array}$ & $\begin{array}{l}\text { 1. Autoinstrucciones antes del } \\
\text { suceso. Fase de preparación } \\
\text { Preocuparse no cambia el problema } \\
\text { Piensa qué has de hacer exactamente } \\
\text { Tú puedes conseguirlo. Es más fácil una } \\
\text { vez que se ha empezado. Estarás bien } \\
\text { No te dejes llevar por pensamientos } \\
\text { negativos. Respira y relájate } \\
\text { 2. Autoinstrucciones al comienzo del } \\
\text { suceso: Fase de confrontación } \\
\text { Cálmate, puede controlarlo } \\
\text { Piensa qué has hecho en otras } \\
\text { ocasiones. Sólo tiene que dar un paso } \\
\text { cada vez. Si no piensas en el miedo no } \\
\text { lo sentiré } \\
\text { Concéntrate en lo que tienes que hacer, } \\
\text { no en el miedo. Esto sólo es una señal } \\
\text { para relajarse. } \\
\text { 3. Autoinstrucciones durante la tarea: } \\
\text { Fase de afrontamiento } \\
\text { Respira profundamente, haz una pausa } \\
\text { y relájate. ¿Cuál es el paso siguiente?. } \\
\text { Concéntrate en él. El miedo es natural, } \\
\text { surge, persiste pero no es peligroso } \\
\text { Esto terminará enseguida, no puede } \\
\text { durar siempre, cosas peores podrían } \\
\text { pasar. He sobrevivido otras veces y a } \\
\text { cosas peores } \\
\text { Concéntrate en lo que estás haciendo } \\
4 . \text { Fase de reforzamiento del éxito } \\
\text { Lo hiciste!!. Conseguiste el objetivo. No } \\
\text { fue tan malo. Lo hice bien. Tus } \\
\text { pensamientos eran peores que la } \\
\text { realidad. La próxima vez será más fácil. } \\
\text { Poco a poco lo conseguirás }\end{array}$ \\
\hline
\end{tabular}

Tomamos problemas similares, reformulándolos, modificando algún elemento, combinándolos o introduciendo elementos auxiliares. En estas condiciones formularíamos de nuevo las preguntas anteriores. Otra opción es descomponer el problema en subproblemas e intentar resolverlos. Al mismo tiempo, trataremos de que el alumno afronte la sensación de estar abrumado, a través de la respiración.

El fin de las sesiones 6, 7 y 8 es que el alumno consiga exponerse a la tarea y a la resolución del problema. Una vez conseguido, iniciamos la fase de ejecución y examen, que ha de realizarse viendo y probando con claridad que los pasos son correctos, registrando todos los cálculos, resaltando los logros intermedios, actuando con orden y con precisión, y, por último, explicando el estado de la ejecución. Con un 
buen control de todo el proceso, el resolutor podrá sacar máximo provecho de sus recursos y supera con más facilidad las posibles dificultades que pueda encontrarse.

Las dos últimas sesiones las dedicamos a la evaluación del proceso de resolución, la evaluación de los esfuerzos realizados y a las autogratificaciones. Esta fase es importante, en cuanto que nos permitirá una revisión del trabajo realizado y la transferencia del conocimiento a situaciones futuras. Aquí tratamos de analizar la consistencia de la solución y del proceso. También es deseable describir esquemáticamente el trabajo realizado, analizando la corrección de cada paso, comparando y evaluando las diferentes estrategias posibles, desde diferentes perspectivas (cognitiva y/o emocional). En definitiva, la meta última sería generalizar la solución y el proceso a otras situaciones .

Paralelamente facilitamos el reforzamiento del éxito, que se traduce en autoverbalizaciones como las que aparecen en la figura3. Nuestro último objetivo será evaluar el proceso en su totalidad, comprobar qué se ha aprendido, reconocer los pequeños beneficios, no menospreciar el progreso gradual, alabar el intento y animar a continuar, sin esperar la perfección. Es preciso revisar las tareas y los ejercicios que se proponen en cada una de las sesiones, emitiendo el 'feedback' oportuno.

En la figura 3 se incluyen algunos interrogantes que operativizan el programa en cada una de sus fases de intervención y según los modelos de partida.

\section{CONSIDERACIONES FINALES}

El dominio afectivo está adquiriendo tal protagonismo en la investigación en didáctica de la Matemática que nos lleva a mantener nuestra hipótesis inicial que relaciona actitudes, creencias y emociones con éxito y fracaso en la enseñanza y en el aprendizaje de las Matemáticas. Su importancia ha sido recogida en la propuesta curricular del MEC (1992, pp. 82): "se considera indispensable que el profesorado sea consciente de la importancia de estos contenidos (actitudinales) como aprendizajes propiamente dichos y para la adquisición de otros de tipo conceptual y procedimental'. Los ingredientes cognitivos y emocionales pueden explicar la situación que se produce cuando un alumno se enfrenta ante un problema de matemática que es incapaz de resolver, y esto es así, tanto en la sensación de alegría ante el éxito, como en la sensación de incapacidad que experimenta cuando el fracaso es continuado. Esta última situación aparece, incluso, en casos en los que los estudiantes tienen las herramientas necesarias, conceptuales y procedimentales que permiten al sujeto resolverlo con éxito. Lo que determinan los sentimientos y las emociones que experimentamos, así como nuestra predisposición a actuar, son las percepciones, las evaluaciones que hacemos, nuestros pensamientos y creencias.

Creemos que las ideas aportadas y la propuesta de un programa de intervención de corte psicopedagógico como el que hemos presentado, pueden ser relevantes tanto en la formación del profesorado como en la del alumnado. Por un lado, al profesorado puede serle de utilidad en la instrucción, en la metodología a emplear y en la enseñanza de estrategias. También, será importante conocer el papel que juegan las emociones en el aprendizaje de los conceptos matemáticos, en la resolución de problemas, así como en la aparición y mantenimiento de fracasos escolares y personales. Estamos convencidos de que el profesor de matemáticas puede ser transmisor, no sólo de herramientas cognitivas, sino de expectativas positivas y de motivación de logro. Podrá ayudar a que el alumno adquiera confianza en él mismo y si lo consigue, tendrá la garantía de haber logrado las más altas metas en materia educativa: el desarrollo 
armónico de la personalidad del alumno, su felicidad y la adaptación en cualquier tipo de ámbito (familiar, escolar, laboral o social). Sólo así habrá contribuido a enfocar su ejercicio profesional hacia una educación integral y una educación para la vida.

Queremos terminar este capítulo tomando prestadas las palabras de Daniel Goleman "las emociones descontroladas pueden convertir en estúpida a la gente más inteligente" (Goleman, 1999, p.44). Necesitamos de la competencia emocional para sacar el máximo provecho de nuestros talentos. La gestión de nuestras emociones y de nuestros impulsos supone pensar con claridad y concentradamente, a pesar de las presiones. También supone gobernar emociones conflictivas y permanecer equilibrados, positivos e imperturbables, aun en los momentos más críticos. En la actualidad no sólo se nos evalúa por nuestra formación o experiencia sino por el modo en que nos relacionamos con nosotros mismos y con los demás, combustible necesario para nuestro buen funcionamiento. $Y$ todo ello, añadimos nosotros, es tan cierto, tanto cuando nos referimos a las competencias personales del alumnado que aprende (y enseña) como a las del profesorado que enseña (y aprende). Apostamos por una educación emocional como respuesta a un déficit en la formación básica.

\section{REFERENCIAS}

BERMÚDEZ, J. La interacción persona-situación: Alternativa teórica de investigación. En J. Bermúdez (Ed.), Psicología de la personalidad. Madrid: Uned. 1986

BISQUERRA, R.. Educación emocional y bienestar. Madrid: CissPraxis. 2001

BLANCO, L. Conocimiento y acción en la enseñanza de las Matemáticas

de profesores de EGB y estudiantes para profesores. Servicio de publicaciones de la Universidad de Extremadura. 1991.

BLANCO, L.J. Concepciones y creencias sobre la resolucion de problemas de estudiantes para profesores y nuevas propuestas curriculares. En Quadrante, Revista Teorica e de Investigacao, 6(2), 1997. pp. $45-65$.

Blanco, L. y Guerrero, E. Actitudes y creencias en la eduación Matemática. En M.C. Penalva, G. Torregrosa y J. Vals (Ed.), Aportaciones de la Didáctica de la Matemática a diferentes perfiles profesionales (pp.121-143). Alicante: Universidad de Alicante. 2002.

CALLEJO, M.L. Un club matemático para la diversidad. Narcea: Madrid.1994.

CAMACHO, M., HERNÁNDEZ, J. Y SOCAS, M.M. Concepciones y actitudes de futuros profesores de secundaria hacia la Matemática y su enseñanza:un estudio descriptivo. En Blanco, L.J. y Mellado, V.: La formación del profesorado de ciencias y matemáticas en España y Portugal. Servicio de Publicaciones Diputación Provincial de Badajoz. 81-97. 1995.

CANo-V indel, A. y Miguel-Tobal, J.J. Emoción y salud. Ansiedad y estrés, 7 (2-3), 2001. 111-121.

CARRILLO, J. Modos de resolver problemas y concepciones sobre la matemática y su enseñanza Metodología de la investigación y relaciones. Servicio de Publicaciones. Universidad de Huelva. 1998.

CAPAFONS, A. y SILVA, F. Manual del cuestionario de autocontrol infantil y adolescente. Madrid: TEA.1986. 
Fernández-Abascal, E. G. y PALmero, F. Emociones y salud. Barcelona. Ariel. 1999.

GalRín, J. Las actitudes en educación. Un estudio sobre la educación matemática. Barcelona: Boixareu Universitaria. 1990.

GolemaN, D. La práctica de la inteligencia emocional. Barcelona: Kairós. 1999.

GÓMEZ-CHACÓN, I. La alfabetización emocional en educación matemática: actitudes, emociones y creencias. UNO. Revista de Didáctica de las Matemáticas. oㅜ 13. 1997.

GÓMEZ-CHACÓN, I. Procesos de aprendizaje en matemáticas con poblaciones de fracaso escolar en contextos de exclusión social: Las influencia safectivas en el conocimientode las matemáticas. En MEC, Premios nacionales de Investigación e Innovación Educativa. Colección Investigación. (pp.333-358). Madrid: Ministerio de educación y Cultura-CIDE. 1999.

GÓMEZ Chacón, I. Matemática emocional. Los afectos en el aprendizaje matemático. Madrid: Narcea. 2000.

GUERRERO, E. y BLANCO, L. Estudio preliminar sobre la influencia de las emopciones y los estilos cognitivos ante la tarea académica. En M.I. Fajardo, M.I. Ruiz, A. Ventura, F. Vicente y J.A. Julve (Ed), Psicología de la educación y formación del profesorado (pp.547-557). Badajoz: Psicoex. 2002.

GuerRero, E., BLANCO, L.J. y VICENTE, F. Trastornos emocionales ante la educación matemática. En García, J.N. (coord.), Aplicaciones para la intervención psicopedagógica. Madrid: Pirámide, 2002

GUZMÁN, M. Para pensar mejor. Barcelona: Labor. 1991.

HANIN, Y.L. A study of anxiety in sport. En W. F. Straub (Ed.), Sport Psychology: An analysis of athlete behavior. Ithaca, N.Y: Mouvemente Publications. 1980.

HARDY, L. A catastrophe model of performance in sport. En J.G. Jones y L. Hardy (Eds), Stress and performance in sport. Chichester: Wiley. 1990.

KERR, J.H. The experience of arousal: A new basis fos studying arousal effects in soprt. Jornal of Sport Sciences, 3. 1985. 169-179.

LANG, M. y TISHER, M. Manual del cuestionario de depresión para niños. Madrid. TEA. 1986.

LAZARUS, R.S. Cognition and motivation in emotion. American Psychologist, 46, 1991. 352-367.

LAZARUS, R.S. y Folkman, S. Psychological stress and the coping process. New York: Mc Graw-Hill. 1976.

LEWIS, M. Y HAVILAND, J.M. Handbook of emotions. Nueva York: Guilford. 1993.

MATTHEW, MC., DAVIS, M. y FANNING, P. Técnicas cognitivas para el tratmiento para el tratmiento del estrés. Madrid: Martínez Roca. 1981.

MEC. Primaria. Area de Matemáticas. 1992.

MeichenbaU, D. Manual de inoculación de estrés. Madrid: Martínez Roca. 1985.

Miguel tobal, J.J., Casado, M.I., CANo Videl, A. Manual del Inventario de situaciones y Respuestas de Ansiedad -ISRA-. Madrid: TEA. 1994.

MIRANDA, A., FORTES, C. y GIL, M.D. Dificultades del aprendizaje de las Matemáticas. Un enfoque evolutivo. Málaga: Aljibe. 1998. 
NewcomB, T. Manual de Psicología Social. Buenos aires: Endeba. 1976.

NeZU, A.M., NeZU, C.M. y BlissetT, S.E. Sense of humor as a moderator of relation between stressful events and psychological distres: A prospective analysis. Journal of Personality and Social Psychology, 54. 1988. 520-525.

OATLEY, K. Y JOHNSON-LAIRD, P.N. UnderstandingEemotions. Cambridge, Ma: Blackwell Pu. 1996.

PORTER, R. B. Y CATELL, R. B. Manual del cuestionario de personalidad para niños. Madrid: TEA.1988.

POYLA, G. Cómo plantear y resolver problemas. México: Trillas. 1985.

PUIG, L. Elementos de resolución de problemas. Granada: Comares. 1996.

SPENCE, J. T. y SPENCE, K.W.The motivational componentess of manifiest anxiety: drive and drive stimuli. En C.D. Spieleberger (Ed.), Anxiety and behavior. New York: Academic Press. 1966.

SPIELBERGER, C.D. y col. Manual del cuestionario de autoevaluación ansiedad estado/rasgo en niños. Madrid: TEA.1990.

SCHOENFELD, A.H. Learning to think mathematically: problem solviing, metacognition, and sesnse-making in mathematics. En D.A.Grouws (Ed.), Handbook of Research on Mathematics teachin and learning (334-370). New York:Mac Millan P.C. 1992.

TOBAL, J.J. Evaluación de las respuestas cognitivas, fisiológicas y motoras de ansiedad: Elaboración de un instrumento de medida. Tesis doctoral. Facultad de psicología de la Universidad Complutense de Madrid. 1985.

YERKES, R.M Y DODSON, J.D. The relation of stregth of stimulus to rapidity of habit-formulation. Journal of Comparative and Neurological Psychology. 18. 1980. 459-482. 